\title{
LBP and Irregular Graph Pyramids
}

\author{
Martin Cerman , Rocio Gonzalez-Diaz , and Walter Kropatsch \\ 1 PRIP Group, Vienna University of Technology, 1040 Wien, Austria \\ mcerman@prip.tuwien.ac.at, rogodi@us.es \\ 2 Applied Mathematics Department, School of Computer Engineering, \\ University of Seville, Seville, Spain \\ krw@prip.tuwien.ac.at
}

\begin{abstract}
In this paper, a new codification of Local Binary Patterns (LBP) is given using graph pyramids. The LBP code characterizes the topological category (local max, min, slope, saddle) of the gray level landscape around the center region. Given a $2 \mathrm{D}$ grayscale image $I$, our goal is to obtain a simplified image which can be seen as "minimal" representation in terms of topological characterization of $I$. For this, a method is developed based on merging regions and Minimum Contrast Algorithm.
\end{abstract}

Keywords: Local binary patterns - Irregular graph pyramid · Primal and dual graph $\cdot$ Topological characterization $\cdot$ Minimum contrast algorithm

\section{Introduction}

Given a grayscale digital image $I$, the Local Binary Pattern $\operatorname{LBP}(I)[12,13]$ is a grayscale digital image used to represent the texture element at each pixel in $I$. This is currently the most frequently used texture descriptor [18] with outstanding results in applications ranging from segmentation and classification [15], object detection [11] to gender classification [16]. Typically the LBP operator is applied to all $3 \times 3$ image windows of the considered texture (region). Then the histogram provides the characteristic features of the texture. After training the feature space with the textures of interest new textures can be classified with very good discrimination.

While the LBP code of a $3 \times 3$ window needs 8 bits for a single code, larger windows need more bits or even a varying number of bits if a multiresolution approach is chosen. The new encoding overcomes this drawback by transferring the code from the pixels to the neighbor relations (edges of the neighborhood

M. Cerman-Acknowledges the support of the PRIP Club, the organization of friends and promoters of Pattern Recognition and Image Processing activities, Vienna, Austria.

R. Gonzalez-Diaz-Author partially supported by IMUS, Spanish Ministry under grant MTM2012-32706 and ESF ACAT program. 
graph). We propose a new equivalent encoding using one bit per edge of the graph.

It is known that the LBP encodes the basic topological categories of a digital image's landscape, e.g. extrema, saddle points, plateaus and slopes. After the contraction of all plateaus, these categories can be identified by simple local detectors except for the saddle points. In a discrete image a saddle can be either an identified pixel or a $2 \times 2$ non-well composed configuration. As the first major novelty introduced in this paper, we show a way to handle also such situations. We further propose a repetitive contraction process leading to hierarchy of successively smaller graphs, a graph pyramid, preserving the basic topological categories in a substantially smaller graph structure.

The second novelty in this paper is the definition of a minimum contrast representative (MCR) image. It stands for the large class of all possible images that produce the same LBP codes and, consequently, also the same LBP histograms. This may allow to understand the trained classifiers since it defines the equivalence class of images of the trained class.

The paper is organized as follows: In Section 2, the topological category of the gray level landscape around a pixel $c$ characterized by its LBP code, is defined. Section 3 recalls irregular graph pyramids. In Section 4, we introduce the notion of well-composed images and explain how any image can be made wellcomposed by inserting a few dummy regions. The goal of the rest of the sections is to obtain a "minimal" representation of a given image in terms of topological characterization. Our idea is to merge pixels (or regions, in general) that provide redundant information, to create the pyramid. The first step in the process is to remove the asymmetry caused by the sign function (see Eq. (1)). For this, in Section 5, we merge regions with same gray value (plateaus). After merging all plateaus, we define the notion of structurally redundant edges that should be removed to reach our goal. In Section 6, we define the topological category of a region. Section 7 is devoted to a particular topological category named singular slope that can be merged without changing the topological category of the rest of the regions. Finally, in Section 8, we adapt Minimum Contrast Algorithm given in [17] to be applied to our pyramid in order to obtain a "minimal" representation of the image. Section 9 is devoted to conclusions, experiments and future works.

\section{Local Binary Patterns (LBP)}

The intensity of a pixel $p=(x, y)$, denoted by $g(p)$, is expressed within a given range between a minimum and a maximum, inclusive. Without loss of generality we will work with 8 bits, i.e., we will suppose that the range is $[0,255]$.

The standard LBP code [12] is computed for a (center) pixel as in Eq. (1) where $P$ is the number of neighbors, $R$ is the distance between the center pixel and the neighbors (we assume 1 here), $c$ is the center pixel of the operator and $p$ is the local neighbor indexed by $p$. The basic operator uses the sign function $s(x)=1$ if $x \geq 0$ and $s(x)=0$ otherwise. 


$$
L B P_{P, R}=\sum_{p=0}^{P-1} s(g(p)-g(c)) 2^{p} .
$$

In this paper, the 4 neighbors (on its top, bottom, right, left) of each pixel are considered for comparison. That is, follow the 4 pixels along a circle (for example, clockwise). Where the center pixel's gray value is greater than the neighbor's gray value, write 0. Otherwise, write 1. Example:

$$
\begin{array}{|c|c|c|}
\hline 113 & 240 & 23 \\
\hline 20 & 25 & 12 \\
\hline 15 & 30 & 40 \\
\hline
\end{array} \Rightarrow \begin{gathered}
1 \\
025 \mathbf{0} \\
1
\end{gathered} \Rightarrow \mathbf{0 1 0 1} \Rightarrow 9
$$

The 4-neighbor LBP codification has been used in the past for solving problems in Image Processing and Analysis such as, for example, face detection and recognition [6], or iris extraction [5].

The LBP code characterizes the topological category of the gray level landscape around the center pixel. A pixel is a local maximum if the LBP code is composed just by 0s. A local minimum produces an LBP code only with 1s. Notice however that a local minimum can also be created by a plateau (a region composed by neighboring pixels sharing the same gray value) due to the asymmetry of the sign function $s$. It is a slope if there is exactly one transition from 0 s to $1 \mathrm{~s}$ or $1 \mathrm{~s}$ to $0 \mathrm{~s}$ in its LBP code. Otherwise, it is a saddle.

\section{LBP Codes and Irregular Graph Pyramids}

A region adjacency graph (RAG) encodes the adjacency of regions in a partition. A vertex $v$ is associated to each region $r$. Vertices of neighboring regions are connected by edges. Classical RAGs do not contain any self-loops or parallel edges. An extended region adjacency graph (eRAG) is a RAG that contains the so-called pseudo edges, which are self-loops and parallel edges used to encode neighborhood relations to a region completely enclosed by one or more other regions [7].

The dual graph of an eRAG $G$ is denoted by $\bar{G}$ ( $G$ is said to be the primal graph of $\bar{G}$ ). The edges of $\bar{G}$ represent the boundaries (borders) of the regions encoded by $G$, and the vertices of $\bar{G}$ represent points where boundary segments meet. $G$ and $\bar{G}$ are planar graphs if they represent a $2 \mathrm{D}$ decomposition into regions. There is a one-to-one correspondence between the edges of $G$ and the edges of $\bar{G}$, which induces a one-to-one correspondence between the vertices of $G$ and the 2D cells (regions) of $\bar{G}$. The dual of $\bar{G}$ is again $G$. The following operations are equivalent: edge contraction in $G$ with edge removal in $\bar{G}$, and edge removal in $G$ with edge contraction in $\bar{G}$. Edge removal preserves the topology (i.e., regions are always homeomorphic to disks) [3].

A (dual) irregular graph pyramid $[7,8]$ is a stack of successively reduced planar graphs $P=\left\{\left(G_{0}, \bar{G}_{0}\right), \ldots,\left(G_{n}, \bar{G}_{n}\right)\right\}$. Each level $\left(G_{k}, \bar{G}_{k}\right), 0<k \leq n$ is 


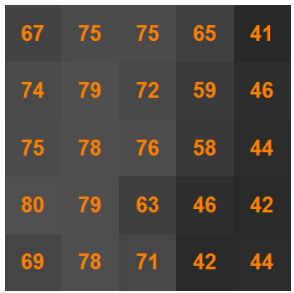

(a) grayscale image

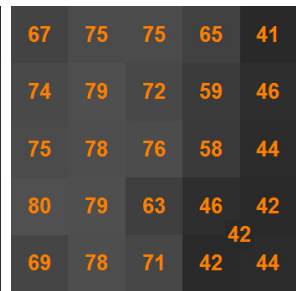

(b) well-composed

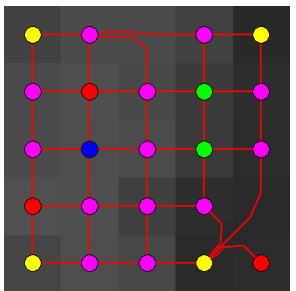

(c) merged plateaus

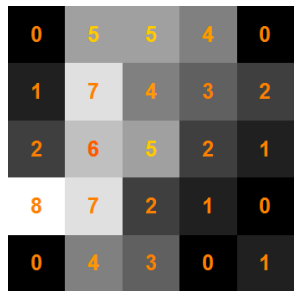

(d) MCR

Fig. 1. An 8-bit grayscale image (highlighted grayscale values) is (b) made wellcomposed. (c) Plateaus are merged (for color legend see Fig. 3(c)) and (d) Minimum Contrast Algorithm produces the minimum contrast representative (MCR in Section 8).

obtained by first contracting edges in $G_{k-1}$ (removal in $\bar{G}_{k-1}$ ), if the corresponding regions should be merged, and then removing edges in $G_{k-1}$ (contraction in $\left.\bar{G}_{k-1}\right)$ to simplify the structure.

In this paper, pixels are considered unit-square regions, 4-neighborhood is used for constructing the RAG and each vertex $v$ of the RAG associated to each region $r$ is labeled with the gray value of the region, i.e., $g(v):=g(r)$.

\section{Creating Well-Composed Images}

A 2D image is well-composed [9] if it does not contain the following non-well composed configuration (modulo reflection and 90-degree rotation):

$$
\begin{array}{|l|l|l|}
\hline a & b \\
\hline c & d
\end{array} \quad \text { where } g(a)<g(b), g(a)<g(c), g(d)<g(b) \text { and } g(d)<g(c) \text {. }
$$

Lemma 1. If the image is well-composed, then the topological category provided by $L B P_{4,1}$ is the same as $L B P_{8,1}$.

Proof. Consider the following $3 \times 3$ block \begin{tabular}{|l|l|l|l|}
\hline$a$ & $d$ & $x$ \\
\hline$b$ & $e$ & $h$ \\
\hline$c$ & $f$ & $i$
\end{tabular}. The topological class of $e$ using $\mathrm{LBP}_{4,1}$ is given by $b, d, f$, and $h . \mathrm{LBP}_{8,1}$ is additionally given by $a, c, x$, and $i$. The topological category of $\mathrm{LBP}_{8,1}$ is the same as $\mathrm{LBP}_{4,1}$, if by adding the diagonal 8-neighbors to $\mathrm{LBP}_{4,1}$ the number of transitions from 0 s to $1 \mathrm{~s}$ or $1 \mathrm{~s}$ to 0 s does not change. In the following we show that any addition of a diagonal element, which would change the topological class, causes the image to become non-well composed (modulo reflection and 90-degree rotation):

- Local minimum (resp. maximum) is defined by $g(e)<g(p)$ (resp. $g(e)\rangle$ $g(p))$ for $p=b, d, f, h$. If $g(e)>g(q)$ (resp. $g(e)<g(q)$ ) for $q=a, c, x$ or $i$, the image becomes non-well composed.

- Slope is defined by either: 


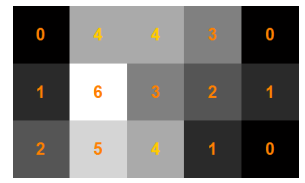

(a) input

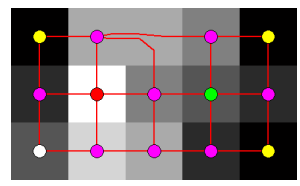

(b) merged plateau

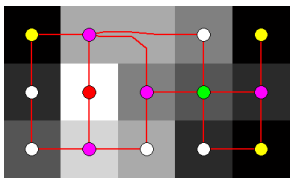

(c) removed edges

Fig. 2. The plateaus of an image with highlighted values are first merged and then structurally redundant edges are removed.

- $g(e)>g(b), g(f), g(h)$ and $g(e)<g(d)$ (resp. $g(e)<g(b), g(f), g(h)$ and $g(e)>g(d))$. If $g(e)<g(c)$ or $g(e)<g(i)$ (resp. $g(e)>g(c)$ or $g(e)>g(i))$ the image becomes not well-composed. Here, $g(a)$ and $g(x)$ are irrelevant.

- $g(e)<g(b), g(d)$ and $g(e)>g(f), g(h)$. If $g(e)>g(a)$ or $g(e)<g(i)$ the image becomes not well-composed. Here, $g(c)$ and $g(x)$ are irrelevant.

- Saddle (resp. plateaus) is defined by $g(e)>g(b), g(h)$ and $g(e)<g(d), g(f)$ (resp. $g(e)=g(b)=g(d)=g(h)$ ). Here $g(a), g(c), g(x)$, and $g(i)$ are irrelevant.

The main problem in a non-well composed configuration like Eq. (2), is that the relation between $a$ and $d$ (resp. $b$ and $c$ ) cannot be deduced from the relation of 4-adjacent regions. To solve this, we insert a new "dummy" region $r$ in the

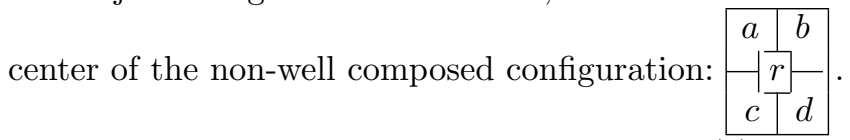

The new region $r$ with a new gray value $g(r)$ reflects the relations between $a, b, c, d$ and in a way that the 8-connectivity LBP code can be deduced from the LBP code of the modified configuration. Without loss of generality, suppose that $g(a) \leq g(d)<g(b) \leq g(c)$. Following cases can occur:

1. $g(a)=g(d)<g(b)=g(c)$ (resp. $g(a)<g(d)<g(b)<g(c))$. In this case, the vertex $v$ represents a saddle. Set $g(r)=\frac{g(d)+g(b)}{2}$. We have that $g(a)=$ $g(d)<\frac{g(b)+g(d)}{2}<g(b)=g(c)\left(\right.$ resp. $\left.g(a)<g(d)<\frac{g(b)+g(d)}{2}<g(b)<g(c)\right)$. Therefore, the LBP code of $r$ is 0101 which is a saddle and all three new vertices have degree 3 and, hence, are well-composed.

2. $g(a)=g(d)<g(b)<g(c)$ (resp. $g(a)<g(d)<g(b)=g(c))$. Set $g(r)=g(d)$ (resp. $g(r)=g(b)$ ). In our process, regions $a, r, d$ (resp. $b, r, c$ ) form a plateau. See Fig. 1(a) and 1(b).

\section{Merging Plateaus and Removing Edges}

The first step in our process to obtain a simplified image with the same topological information as the original, is to merge plateaus. This way, by contracting adjacent vertices with same value in the primal graph (i.e., merging neighboring 


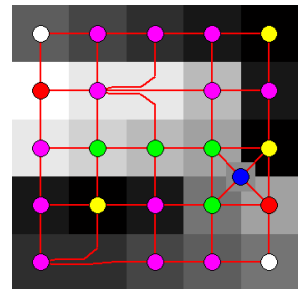

(a) after merging plateaus

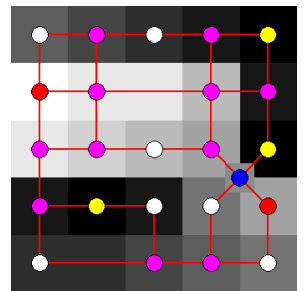

(b) after removing edges

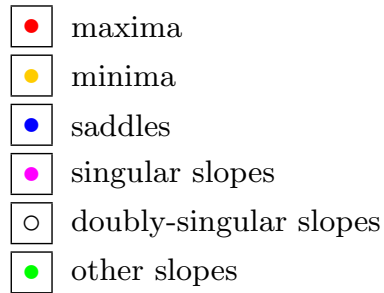

(c) vertex colors

Fig. 3. Removing structurally redundant edges from the primal graph.

regions with same value in the dual), we remove the asymmetry caused by the sign function. See Fig. 2(b).

After merging all plateaus, a direction can be associated to each non-self-loop edge between different vertices of the primal graph $G$ :

$$
(u, v) \in E \text { has direction } u \rightarrow v \text { iff } g(u)>g(v) .
$$

Proposition 1. After merging plateaus, $G \backslash\{$ self-loops $\}$ is a directed graph such that: (a) $G$ does not contain directed cycles. (b) Vertices in $\bar{G}$ do not increase degrees.

Proof. (a) Suppose that $G$ has a directed cycle $u_{0} \rightarrow u_{1} \rightarrow \cdots \rightarrow u_{n}=u_{0}$. Then $u_{0}>u_{1}>\cdots>u_{n}=u_{0}$, which is a contradiction. (b) Merging plateaus in $G$ consists of removing edges and merging vertices of degree $\leq 2$ in $\bar{G}$. Therefore, vertices in $\bar{G}$ have degree 3 or 4 .

An oriented edge $(u, v) \in E$ is considered "structurally redundant" if there exists a dual vertex $\bar{w} \in \bar{V}$ bounded by $(u, v)$ and a directed path $p(u, v)$ from $u$ to $v$. Structurally redundant edges can be removed in $G$ (see Fig.2(c) and 3(b)).

Finally, the notion of well-composed configurations can be extended to regions in $\bar{G}$ due to Prop. $1(\mathrm{~b})$.

\section{Topological Category of Regions}

After merging plateaus, in the same way as for pixels, we can define the topological category of a vertex $v$ of $G$ by considering the edges incident to $v$. See Fig. 1(c) and 3(a). Following the edges incident to $v$ :

- $v$ is a local minimum if it is the head of all the edges incident to it.

- $v$ is a local maximum if it is the tail of all the edges incident to it.

- $v$ is a doubly-singular slope if it has degree two and it is the tail of one of the edges incident to it and the head of the other.

- $v$ is a singular slope if it has degree greater than two and it is the tail (resp. head) of exactly one edge incident to it and the head (resp. tail) of the others.

- $v$ is a slope if $v$ is the tail of the first edges incident to it and the head of the others, clockwise or counter-clockwise.

$-v$ is a saddle otherwise. 


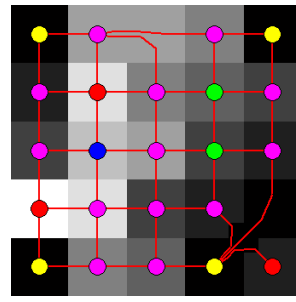

(a) graph -plateaus

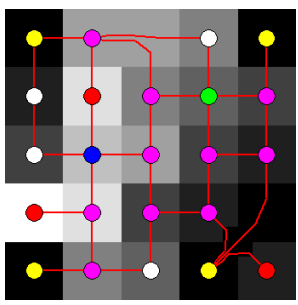

(b) -redundant edges

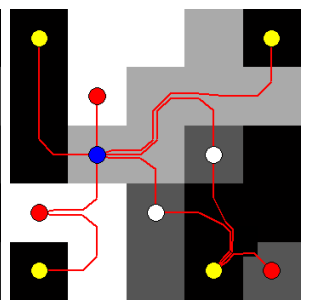

(c) -singular slopes

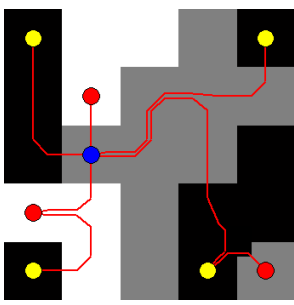

(d) minimal graph

Fig. 4. The primal graph $G$ is successively reduced by merging plateaus and (doubly)singular slopes. Merge operations and removal of structurally redundant edges are applied in an alternating fashion. The last image is considered "minimal".

Proposition 2. After removing redundant edges, the topological category of the vertices of $G$ may be simplified: singular slopes may change to doubly-singular slopes, and slopes to singular slopes (see Fig. 2(c), 3(b) and 4(b)). Nevertheless, local maxima, minima and saddles are always preserved in the primal graph.

Proof. For each structurally redundant edge $e=(u, v) \in E$ there exists a monotonic path connecting $u$ to $v$. Therefore the removal would not disconnect an extremum. And since it is monotonic, the extremum remains extremum.

Assume the endpoint $v$ of a structurally redundant edge $e=(u, v)$ is a saddle. The last directed edge $q=\left(v_{k}, v\right)$ of the alternative path $p(u, v)=\left(u, x_{0}, \ldots, q\right)$ must have the same orientation as $(u, v)$ due to the monotonicity of the path. Since the bounded dual cell $\bar{w}$ does not contain any hole both $e$ and $q$ succeed each other at the saddle $v$. Since they both are either below or above the saddle the removal of $e$ would not change the property of the saddle.

\section{$7 \quad$ Merging Singular Slopes}

After merging plateaus, a direction is associated to the edges of the primal graph and a topological category to its vertices. Structurally redundant edges are then removed and the topological category of the vertices is updated.

Further steps in our process to obtain a simplified image with same topological information as the original, should remove topologically redundant information by merging regions. In general, a region obtained after merging slopes around a local maximum (resp. minimum) is not a local maximum (resp. minimum). Singular slopes make an exception. Merging singular slopes propagates well around local extrema since a local extremum in the surrounding regions often is or becomes a singular slope. However non-well composed configurations (corresponding to saddles in the dual graph) can block propagation. This is why we insert dummy regions in non-well composed configurations.

Prop. 3 asserts that contracting a singular slope $p$ to a vertex $q$ in the primal graph, does not change the topological category of $q$. 
Proposition 3. Consider a vertex $p$ which is a singular slope in the primal graph. Let $E$ be the set of edges incident to $p$. Let $p$ be the head (resp. tail) of exactly one edge $e \in E$ and it is the tail of the rest. Let $q$ be the tail (resp. head) of $e$. Then the vertex obtained after contracting $p$ to $q$ (i.e., after merging the two associated regions in the dual graph) inherits the topological category of the region q, i.e. local max, min, saddle or slope.

Proof. After contracting $p$ to $q$, the edge $e$ is replaced by the set of edges $E \backslash e$ in such a way that $q$ is the tail (resp. head) of all these edges. Since $q$ was the tail (resp. head) of $e$, then, the topological category of $q$ does not change. There is no inconsistency in the new graph: Let $w$ be the head (resp. tail) of one edge in $E \backslash e$. Then $g(w)<g(p)<g(q)$ (resp. $g(w)>g(p)>g(q)$ ), therefore $g(w)<g(q)$ (resp. $g(w)>g(q)$ ), e.g. no new oriented cycle has been generated.

Conjecture 1. The result of merging all singular slopes does not depend on the order we merge.

Observe that no new singular slopes can appear after merging singular slopes, since the topological category of the rest of the vertices remains invariant. But, new structurally redundant edges can appear. Therefore, the removal of structurally redundant edges and merging singular slopes can be repeated until no more reductions are possible. Finally, surviving slopes are merged to saddles. This way, we obtain an irregular graph pyramid. On the top of the pyramid, only local maxima, minima and saddles can appear. Besides, the number of local maxima, minima and saddles of the original and reduced image coincide (see Fig. 4).

Note that after merging a singular slope $p$, no "corner" adjacent regions with same gray values could become adjacent later since it would mean that $p$ would have at least two 0s in its LBP code. Besides, as a result after contracting plateaus, the reconstructed LBP code is defined for regions thus having as many bits as adjacent regions. Moreover, after merging all plateaus, the LBP codes are symmetric, and, the operations "image complement" and "LBP code" for regions are commutative. Finally, observe that after merging all singular slopes, each slope has at least two 1s and two 0s in its LBP code.

\section{Reconstruction - Representative Image}

In [17] and much earlier in [10], the authors leverage an inverse problem approach to show that it is possible to directly reconstruct the image content from Local Binary Patterns. For this aim, ascending and descending monotonic paths are considered. Their algorithm, $\mathrm{MCA}^{1}$ assumes a minimum contrast of one between two successive pixels; and reconstructs some of the contrast lost in the LBP process. Fig. 5(b) illustrates MCA: after initializing the result with 0 , neighbors which should be higher $($ red $>$ ) are repeatedly incremented until all inequalities

\footnotetext{
${ }^{1}$ Minimum Contrast Algorithm.
} 


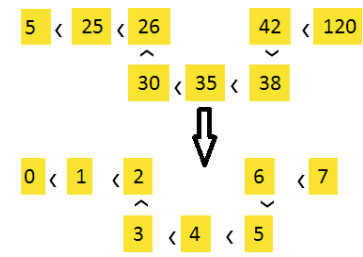

(a)

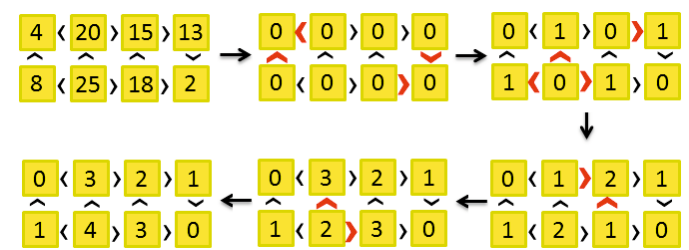

(b) MCA converts a digital image to a MCR.

Fig. 5. (a) A monotonic ascending path with same inequalities than a minimum contrast path. (b) A naive illustration of MCA.

are satisfied. This problem of reconstructing images from features is also dealt in [1] in which the image is encoded using LBD (Local Binary Descriptors) codes which are related but different to LBP codes.

After merging plateaus and singular slopes and removing structurally redundant edges, our image is decomposed in not-necessarily squared regions. In this section we show that the method explained in [17] can be translated in terms of primal and dual graphs. The key is that LBP categories of the $\mathrm{MCR}^{2}$ must be equal to those of original image. For this, longest monotonically increasing (decreasing) paths from local minima (maxima) are computed.

Definition 1. Given an image I, a monotonic ascending path is a sequence $p_{1}, p_{2}, \ldots, p_{n}$ of regions such that: (1) $p_{i}$ is adjacent ${ }^{3}$ to $p_{i-1}$ and (2) $g\left(p_{i-1}\right)<$ $g\left(p_{i}\right)$, for $1<i \leq n$. Whenever $g\left(p_{i-1}\right)>g\left(p_{i}\right)$, for $1<i \leq n$, the path is called monotonic descending (see Fig. 5(a)).

To simulate MCA on our graph after contracting plateaus we initialize all vertices with 0 . We then repeat following update operation for every oriented edge $(u, v)$ : replace $g(v)$ by the maximum of $g(v)$ and $g(u)+1$ until no further change occurs. When starting with maxima we initialize with 255 and repeatedly replace $g(u)$ by the minimum of $g(u)$ and $g(v)-1$. In Fig. 1(d) a naive example of the application of MCA is given.

This process opens a door to what we call representative image. Let $I$ be an image and $L B P(I)$ its associated image of LBP codes. There is a whole set of images $\rho(I)$ for which the LBP codification is identical. Let $L B P_{\min }^{-1}(I)$ be the image obtained after applying MCA on all monotonic ascending paths, and $L B P_{\max }^{-1}(I)$ be the image obtained from all monotonic descending paths. Then both $L B P_{\min }^{-1}(I)$ and $L B P_{\max }^{-1}(I)$ can be used to represent $\rho(I)$. In particular $L B P_{\text {min }}^{-1}(I) \in \rho(I)$ and it is the smallest $L B P_{\text {min }}^{-1}(I) \leq J$ for all $J \in \rho(I)$. Analogously, $L B P_{\max }^{-1}(I) \in \rho(I)$ and it is the largest $L B P_{\max }^{-1}(I) \geq J$ for all $J \in \rho(I)$. Fig. 6 shows the original image $I$ and the two representative images. Notice, how visually different these images from the same LBP class may look.

Proposition 4. For any two images $I, J \in \rho(I), \quad I \neq J$ the property $L B P_{\min }^{-1}(I)=L B P_{\min }^{-1}(J)$ and $L B P_{\max }^{-1}(I)=L B P_{\max }^{-1}(J)$ holds.

\footnotetext{
${ }^{2}$ Minimal Contrast Representative.

${ }^{3}$ In the image we assume 4-adjacency while edges encode adjacency in the graph.
} 

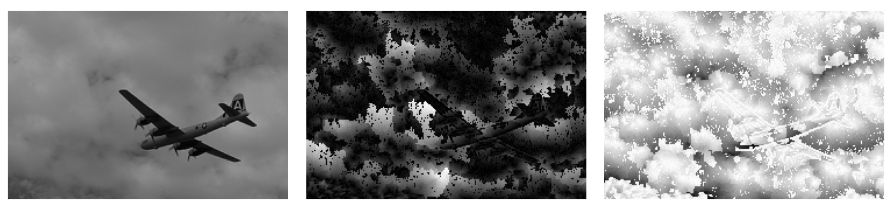

Fig. 6. From left to right: The original image; Reconstructed image by MCA on monotonic ascending paths; Reconstructed image by MCA on monotonic descending paths.
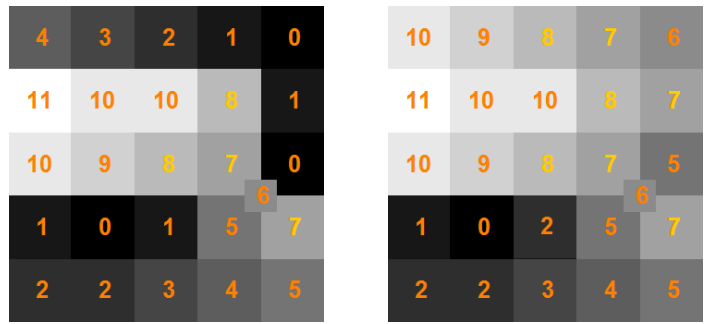

Fig. 7. Image $A$ on the left is an output of the current MCA. Image $B$ on the right has the property $L B P(B)=L B P(A)$, and also has a lower total contrast than $A$.

Proof. The direction of each edge $(u, v)$ remains the same during the computation process of the representative images. Starting points for generating $L B P_{\min }^{-1}(I)$ and $L B P_{\max }^{-1}(I)$ are well defined, namely all local minima and all local maxima. The minimum contrast value of each region is defined by the longest distance from any reachable starting point, from which follows that the order of propagation of the minimum contrast values does not matter.

\section{Conclusion, Experiments and Future Works}

In this paper, a new codification of Local Binary Pattern is given using graph pyramids. For well-composed images, we demonstrate that from 4 neighbors' topological information, we can obtain 8 neighbors topological information what makes classical 8-neighbor LBP topologically redundant. By inserting a few dummy regions every image can be made well-composed! A sequence of merging regions and removal of edges obtains a "minimal" image $I$ with the same topological information as the original. $\mathrm{MCA}$ is adapted ${ }^{4}$ to obtain a minimum contrast image from $I$.

In Fig. 8, the whole process on two grayscale images is shown. After merging plateaus and removing redundant edges, (doubly-)singular slopes are merged successively. After each merging, redundant edges are removed. Finally, if slopes survive, they are merged with saddles. Therefore, only local maxima, minima and saddles survive on the top of the pyramid.

\footnotetext{
${ }^{4}$ A preliminary version of Section 8 was presented in [4].
} 


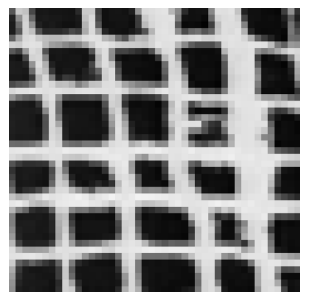

(a) original image

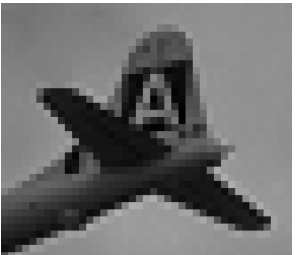

(e) original image

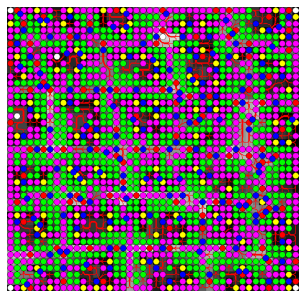

(b) -plateaus

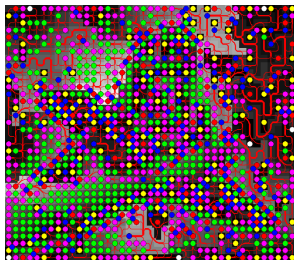

(f) -plateaus

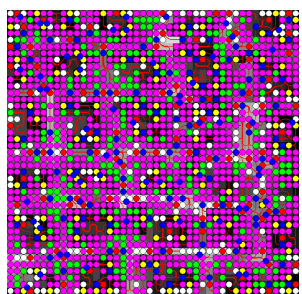

(c) -redundant edges

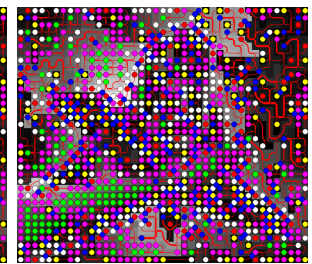

(g) -redundant edges

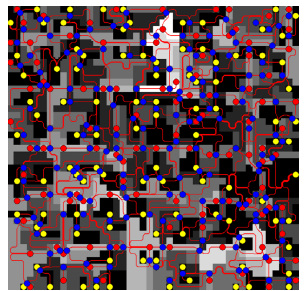

(d) minimal graph

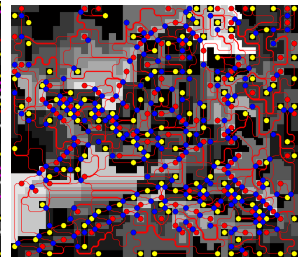

(h) minimal graph

\begin{tabular}{|c||c|c|c|c|c|c|}
\hline Image caption & maxima & minima & saddles & slopes & singular slopes & doubly-singular slopes \\
\hline (b) & 109 & 103 & 179 & 625 & 693 & 7 \\
(c) & 109 & 103 & 179 & 196 & 971 & 158 \\
(d) & 109 & 103 & 179 & 0 & 0 & 0 \\
\hline (f) & 134 & 131 & 224 & 359 & 361 & 6 \\
(g) & 134 & 131 & 224 & 108 & 404 & 214 \\
(h) & 134 & 131 & 224 & 0 & 0 & 0 \\
\hline
\end{tabular}

Fig. 8. The original images are reduced first by merging plateaus and second by removing structurally redundant edges. After removing all (doubly-)singular slopes, regular slopes are merged with saddles. Finally, only local maxima, minima and saddles survive.

"Truly" Minimum Contrast Image. The MCA can be improved to minimize the total contrast $\sum_{(u, v) \in E} g(u)-g(v)$. See, for example, Fig. 7. This approach may start by initializing the absolute minimum to 0 or an arbitrary vertex to an arbitrary value, and subsequently use the sign of the contrast associated at the edges to compute the minimal contrast representative.

Image Segmentation. Modifications made to the proposed approach in form of attributed vertices and weighted edges allow the definition of texture aware image segmentation algorithms. A first attempt made in this direction was presented in [19].

Towards 3D. Several researchers have been trying to extend the LBP codification from $2 \mathrm{D}$ plane to $3 \mathrm{D}$ volume (see, for example, $[2,14]$ ); however, it is not so straightforward as it appears at first glance. In our case the notion of well-composed images and irregular graph pyramids also works for 3D images. Characterizations of pixels using LBP codes not only depend on the number of connected components but also on the number of holes (1-dimensional homology classes). 
Shape LBP. Textures have particular shapes. The idea is to label the darts as 0 or 1 in the dual graph depending on concavity-convexity of the boundary of a region. Computing LBP codification using concavity-convexity rule could help to recognize shapes.

\section{References}

1. D'Angelo, E., Jacques, L., Alahi, A., Vandergheynst, P.: From Bits to Images: Inversion of Local Binary Descriptors. IEEE Transactions on Pattern Analysis and Machine Intelligence 36(5), 874-887 (2014)

2. Fehr, J., Burkhardt, H.: 3D rotation invariant local binary patterns. In: Proc. of 19th Int. Conf. on Pattern Recognition (ICPR 2008), pp. 1-4 (2008)

3. Gonzalez-Diaz, R., Ion, A., Iglesias-Ham, M., Kropatsch, W.G.: Invariant representative cocycles of cohomology generators using irregular graph pyramids. Computer Vision and Image Understanding 115(7), 1011-1022 (2011)

4. Gonzalez-Diaz, R., Kropatsch, W.G., Cerman, M., Lamar, J.: Characterizing configurations of critical points through LBP. In: Int. Conf. on Computational Topology in Image Context (CTIC 2014). Extended Abstract. http://www.prip.tuwien. ac.at/people/krw/more/papers/2014/CTIC/Gonzalez2014b.pdf

5. Guo, G., Jones, M.J.: Iris extraction based on intensity gradient and texture difference. In: Proc. of the 2008 IEEE Workshop on Applications of Computer Vision (WACV 2008), pp. 1-6. IEEE Computer Society, Washington, DC (2008)

6. Hadid, A., Pietikäinen, M., Ahonen, T.: A discriminative feature space for detecting and recognizing faces. In: Proc. of the 2004 IEEE Computer Society Conf. on Computer Vision and Pattern Recognition (CVPR 2004), pp. 797-804. IEEE Computer Society, Washington, DC (2004)

7. Kropatsch, W.G.: Building irregular pyramids by dual-graph contraction. IEEE Proc. on Vision Image and Signal Processing 142(6), 366-374 (1995)

8. Kropatsch, W.G., Haxhimusa, Y., Pizlo, Z., Langs, G.: Vision pyramids that do not grow too high. Pattern Recognition Letters 26(3), 319-337 (2005)

9. Latecki, L., Eckhardt, U., Rosenfeld, A.: Well-composed sets. Computer Vision and Image Understanding 61, 70-83 (1995)

10. Lindahl, T.: Study of Local Binary Patterns. Master's thesis, Linköpings Tekniska Högskola (2007)

11. Nguyen, D.T., Ogunbona, P.O., Li, W.: A Novel Shape-based Non-redundant Local Binary Pattern Descriptor for Object Detection. Pattern Recognition 46(5), 1485-1500 (2013)

12. Ojala, T., Pietikainen, M., Harwood, D.: A comparative study of texture measures with classification based on featured distributions. Pattern Recognition 29(1), 51-59 (1996)

13. Ojala, T., Pietikainen, M., Maenaa, T.: Multiresolution Gray-scale and Rotation Invariant Texture Classification with Local Binary Patterns. IEEE TPAMI 24(7), 971-987 (2002)

14. Paulhac, L., Makris, P., Ramel, J.-Y.: Comparison between 2D and 3D local binary pattern methods for characterisation of three-dimensional textures. In: Campilho, A., Kamel, M.S. (eds.) ICIAR 2008. LNCS, vol. 5112, pp. 670-679. Springer, Heidelberg (2008)

15. Pietikäinen, M., Hadid, A., Zhao, G., Ahonen, T.: Computer Vision Using Local Binary Patterns, vol. 40. Springer Verlag (2011) 
16. Shan, C.: Learning Local Binary Patterns for Gender Classification on Real-world Face Images. Pattern Recognition Letters 33(4), 431-437 (2012)

17. Waller, B.M., Nixon, M.S., Carter, J.N.: Image reconstruction from local binary patterns. In: IEEE proc. of the 2013 Int. Conf. on Signal-Image Technology \& Internet-Based Systems (SITIS), pp. 118-123 (2013)

18. LBP 2014 Workshop on Computer Vision With Local Binary Pattern Variants. https://sites.google.com/site/lbp2014ws/

19. Cerman, M.: Structurally Correct Image Segmentation using Local Binary Patterns and the Combinatorial Pyramid. Technical Report 133, Vienna University of Technology, Pattern Recognition and Image Processing (PRIP) Group (2015). ftp://ftp.prip.tuwien.ac.at/pub/publications/trs/tr 